\title{
Body composition in prostate cancer patients: novel insights suggest diverse prognostic roles of lean and fat mass
}

\author{
Jacob Uth $^{1} \cdot$ Jesper Frank Christensen ${ }^{2}$
}

Received: 12 May 2015/Accepted: 25 May 2015/Published online: 4 June 2015

(C) Springer Science+Business Media New York 2015

Few advances have led to such profound progress in clinical oncology as the pioneering work by Huggins et al. who unveiled the therapeutic potential of surgical/chemical castration in the control of prostatic adenocarcinoma more than 70 years ago [1]. By lowering circulating androgen levels, most importantly testosterone, cancer-specific outcomes are markedly improved, and today, androgen deprivation therapy (ADT) is a mainstay in prostate cancer management. However, ADT-induced hypogonadism causes a plethora of adverse reactions, which may predispose to poor clinical outcomes. In particular, increase in whole-body and visceral fat mass with concomitant progressive loss of lean body mass and impaired bone mass density (BMD) have been extensively documented over the last decade [2]. These unfavorable changes can lead to a high incidence of osteoporosis and bone fractures [3] and are associated with increased risk of Type 2 Diabetes Mellitus and cardiovascular disease observed in men undergoing ADT [4].

In this issue of Endocrine, Buttigliero et al. [5] report body composition changes in 53 patients [mean age 71 years (range 44-83) and mean BMI $25 \mathrm{~kg} \mathrm{~m}^{-2}$ (range 19-39)] over the course of 2 years of ADT. Furthermore, the authors followed the subjects for a median of 7.1 years, until death or final clinical follow-up, to explore associa-

Jesper Frank Christensen

jesper.frank.christensen@ regionh.dk

1 The University Hospitals Centre for Health Research (UCSF), Rigshospitalet, University of Copenhagen, Blegdamsvej 9, 2100 Copenhagen, Denmark

2 The Centre of Inflammation and Metabolism and the Centre for Physical Activity Research (CIM/CFAS), Department of Infectious Diseases, Rigshospitalet, University of Copenhagen, Blegdamsvej 9, 2100 Copenhagen, Denmark tions between body composition changes and overall survival (OS), progression-free-survival (PFS), and time-toskeletal-related-events (TTSRE), respectively. The authors show that patients with prostate cancer undergoing ADT experience a progressive, significant decrease in BMD at the lumbar spine of approx. $-0.02 \mathrm{~g} \mathrm{~cm}^{-2}(p<0.03)$, a mean loss of approx. $-0.9 \mathrm{~kg}$ lean body mass $(p<0.03)$, and a mean gain of approx. $+2.2 \mathrm{~kg}$ fat mass $(p<0.0001)$ over the initial 2 years of treatment.

To explore the prognostic role of body composition, the authors present the following outcome measures: (I) baseline levels (pre-ADT); (II) changes over the first year of treatment; and (III) progressive change from first year to second year of treatment, and investigates their predictability of OS, PFS, and TTSRE. A high gain of fat mass during the first year of treatment was associated with a 2.4fold increase in overall mortality risk (HR 2.4, $95 \% \mathrm{CI}$ $1.0-5.6, p=0.04)$ and a threefold higher risk of a skeletalrelated events (HR 3.0, $95 \%$ CI 1.0-10.4, $p=0.02$ ). During the same period, a borderline significant association was observed between a large decrease in lean mass and time to tumor progression (HR 1.6, $95 \%$ CI 0.7-3.6, $p=0.06$ ). In contrast, neither baseline levels nor changes occurring over the second year of treatment in any of the body composition measures were associated with prostate cancer outcomes.

As outlined by the authors, these findings are based on a small-scaled, non-controlled (no non-ADT or healthy control groups) study using unplanned post hoc analyses, and thus should be interpreted with care. But despite these limitations, this investigation extends upon the current evidence describing the sequalae of treatment-related changes in body composition in the oncology setting. A considerable number of studies have explored associations between body composition and cancer outcomes, but 
primarily as cross-sectional/single time-point investigations, which may under- or over-estimate the role of these factors due to intra-individual variation [6]. A major strength of the present study is the report of individual trajectories over time as a direct measure of treatmentinduced impairments as well as their prognostic value in prostate cancer survivorship. Interestingly, the study indicates that changes in lean mass are associated with the risk of disease recurrence, but have little impact in relation to OS in this group; while increased fat mass conversely may predict OS (and surprisingly bone fracture risk), but does not correlate to tumor progression.

This apparently diverse prognostic role of body composition changes in prostate cancer warrants further exploration, and in particular a better understanding of the responsible biological mechanisms. It is generally acknowledged that obesity, in particular characterized by excessive visceral adipose tissue, is associated with increases in systemic adipokines, inflammatory cytokines, glucose, insulin, and sex hormones. In particular adipocytederived circulating androgens can have proliferating actions in prostate adenocarcinomas and have therefore been proposed as a primary candidate responsible for the inverse relationship between obesity and prostate cancer risk, and the subsequent risk of recurrence and mortality [7]. However, the present study indicates that increased fat mass is not directly linked to prostate cancer progression, but is more likely to be associated with general metabolic dysfunction which may facilitate the onset or progression of other morbidities (e.g., diabetes and cardiovascular diseases) as the primary causes of mortality. Conversely, the study indicates there may be a direct association between evidence of muscle dysfunction and tumor progression. Recent studies have pointed out that low muscle mass (sarcopenia) independent of fat- and total body mass, constitutes an important cancer-specific [8] and overall [9] mortality risk factor. The link between muscle function and tumor outcomes has not been fully elucidated, yet preclinical studies have shown a direct anti-proliferative muscle-to-cancer crosstalk mediated through a contractioninduced release of peptides, known as myokines [10]. Further, high level of lean mass improves whole-body insulin sensitivity and overall metabolic control, which may regulate tumor substrate availability and/or metabolism [10].

Despite the emerging evidence underlining the poor clinical outcomes associated with fat gain and loss of lean mass in prostate cancer patients undergoing ADT, no standardized therapeutic countermeasures exist in clinical practice. Different life-style interventions have been shown to effectively improve body composition, but recommendations are equivocal. Based on the present findings, lifestyle interventions should likely have dual focus on both controlling energy turnover by reducing dietary energy intake and increasing physical activity energy expenditure, as well as maintaining skeletal muscle function through structured and more strenuous exercise programs, e.g., with high intensity interval training and progressive resistance training. To this end, there is a need for future research to identify valid biomarkers mediating life-style interventions effects on prostate cancer outcomes which can help clinicians to prescribe, monitor, and adjust actions for prostate cancer patients. With modern advances in personalized medicine, cancer management is moving rapidly towards more individualized care. In support, the study by Buttigliero et al. suggests that change in body composition is a diverse adverse reaction to ADT which may be mediated through different pathways, thus presenting patients with an individual risk profile, from which interventions may be tailored to optimize their protective potential and improve survivorship.

Acknowledgments The Centre of Inflammation and Metabolism (CIM) is supported by a grant from the Danish National Research Foundation (DNRF55). The Centre for Physical Activity Research (CFAS) is supported by a grant from TRYGFONDEN. JFC is supported by a grant from The Danish Cancer Society.

Disclosures The authors have nothing to disclose.

\section{References}

1. C. Huggins, C.V. Hodges, Studies on prostatic cancer. I. The effect of castration, of estrogen and of androgen injection on serum phosphatases in metastatic carcinoma of the prostate. Cancer Res. 1, 293-297 (1941)

2. P.L. Nguyen, S.M. Alibhai, S. Basaria, A.V. D'Amico, P.W. Kantoff, N.L. Keating, D.F. Penson, D.J. Rosario, B. Tombal, M.R. Smith, Adverse effects of androgen deprivation therapy and strategies to mitigate them. Eur. Urol. 67, 825-836 (2015)

3. S.M. Alibhai, M. Duong-Hua, A.M. Cheung, R. Sutradhar, P. Warde, N.E. Fleshner, L. Paszat, Fracture types and risk factors in men with prostate cancer on androgen deprivation therapy: a matched cohort study of 19,079 men. J. Urol. 184, 918-923 (2010)

4. N.L. Keating, A.J. O’Malley, M.R. Smith, Diabetes and cardiovascular disease during androgen deprivation therapy for prostate cancer. J. Clin. Oncol. 24, 4448-4456 (2006)

5. C. Buttigliero, F. Vana, V. Bertaglia, F. Vignani, C. Fiori, G. Osella, F. Porpiglia, M. Tucci, G.V. Scagliotti, A. Berruti, The fat body mass increase after adjuvant androgen deprivation therapy is predictive of prostate cancer outcome. Endocrine (2015). doi:10.1007/s12020-015-0525-x

6. J.F. Christensen, L.W. Jones, J.L. Andersen, G. Daugaard, M. Rorth, P. Hojman, Muscle dysfunction in cancer patients. Ann. Oncol. 25, 947-958 (2014)

7. A. McTiernan, Mechanisms linking physical activity with cancer. Nat. Rev. Cancer 8, 205-211 (2008)

8. C.M. Prado, J.R. Lieffers, L.J. McCargar, T. Reiman, M.B. Sawyer, L. Martin, V.E. Baracos, Prevalence and clinical implications of sarcopenic obesity in patients with solid tumours of the 
respiratory and gastrointestinal tracts: a population-based study. Lancet Oncol. 9, 629-635 (2008)

9. A. Villaseñor, R. Ballard-Barbash, K. Baumgartner, R. Baumgartner, L. Bernstein, A. McTiernan, M.L. Neuhouser, Prevalence and prognostic effect of sarcopenia in breast cancer survivors: the HEAL Study. J. Cancer Surviv. 6, 398-406 (2012)
10. L. Pedersen, J.F. Christensen, P. Hojman, Effects of exercise on tumor physiology and metabolism. Cancer J. 21, 111-116 (2015) 